\title{
Coronary compression by supposed cardiac hydatid cyst: an unusual cardiac mass
}

\author{
Fatih Levent ${ }^{1}$, Efe Edem $^{1}$, Sadık Volkan Emren ${ }^{2}$, Sedat Altay ${ }^{3}$ \\ ${ }^{1}$ Department of Cardiology, Tınaztepe Hospital, Izmir, Turkey \\ ${ }^{2}$ Department of Cardiology, Atatürk Training and Research Hospital, İzmir Katip Çelebi University, Izmir, Turkey \\ ${ }^{3}$ Department of Radiology, Atatürk Training and Research Hospital, İzmir Katip Çelebi University, Izmir, Turkey
}

Adv Interv Cardiol 2018; 14, 2 (52): 208-209

DOI: https://doi.org/10.5114/aic.2018.76418

A 55-year-old female patient with a history of hepatic hydatid cyst presented with chronic stable angina. Echocardiography showed a cardiac mass. Her technetium-99m stress test was positive, so it was decided to perform coronary angiography (CAG). Coronary angiography performed on the next day revealed a chronic total
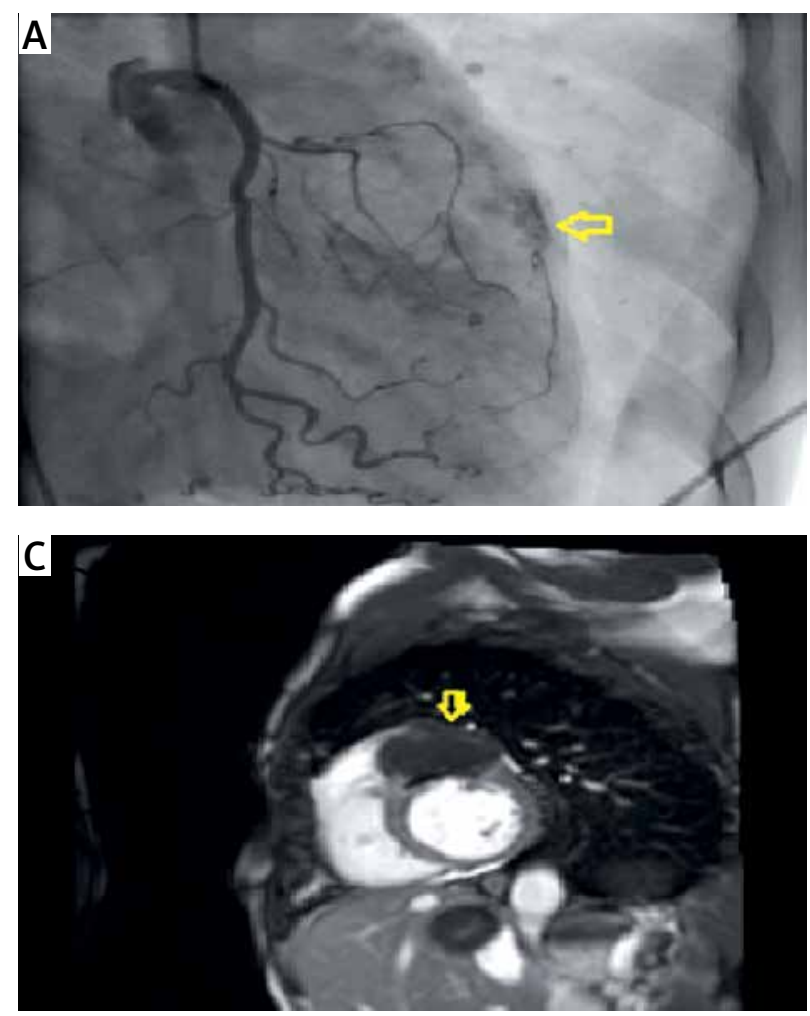

occlusion of the proximal left anterior descending artery (LAD) with retrograde filling via collaterals from the right coronary artery (Figure $1 \mathrm{~A}$ ). Cardiac magnetic resonance imaging (MRI) and multislice computed tomography (CT) angiography confirmed a myocardial hydatid cyst which involved the anterobasal wall of the left ventricle

Figure 1. A - Left coronary angiography (right anterior oblique caudal view) demonstrating chronic total occlusion of the left anterior descending artery (LAD) and a mass next to the LAD (arrow). B - 3D computed tomography image showing the hydatid cyst (arrow). C, D - Magnetic resonance images of the hydatid cyst (arrows) in short axis 
and compressed the proximal LAD (Figures 1 B-D). An enzyme-linked immunosorbent assay (ELISA) was performed to confirm the diagnosis and it was positive for Echinococcus antibodies. The patient was offered surgery but she refused it. We initiated albendazole $800 \mathrm{mg} /$ day and also metoprolol $100 \mathrm{mg} /$ day and trimetazidine $60 \mathrm{mg} /$ day to reduce ischemia and symptoms. At the 3-month follow-up visit, she was almost asymptomatic. However, her follow-up cardiac MRI showed no reduction of the cyst size.

Hydatid disease (cystic echinococcosis) is a rare parasitic infestation caused by the metacestode stage of Echinococcus granulosus that usually involves the liver and lungs [1]. Cardiac involvement is very rare (0.5-2\%). Although most patients with a cardiac hydatid cyst are asymptomatic, large myocardial hydatid cysts may compress the surrounding heart muscle and cause myocardial ischemia [2]. Echocardiography, CT and cardiac MRI are sensitive for diagnosis of cardiac hydatid cyst. Surgical excision is the preferred treatment $[3,4]$.

In conclusion, cardiac hydatid disease, although very rare, should be considered in the differential diagnosis of chest pain and myocardial ischemia, particularly in patients with a prior history of cystic echinococcosis.

\section{Conflict of interest}

The authors declare no conflict of interest.

\section{References}

1. Patkowski W, Krasnodebski M, Grat M, et al. Surgical treatment of hepatic Echinococcus granulosus. Gastroenterology Rev 2017; 12: 199-202.

2. Karadede A, Alyan O, Sucu M, et al. Coronary narrowing secondary to compression by pericardial hydatid cyst. Int I Cardiol 2008; 123: 204-7.

3. Shehatha J, Alward M, Saxena P, et al. Surgical management of cardiac hydatidosis. Tex Heart Inst J 2009; 36: 72-3.

4. Travin N, Shevchenko Y. Heart echinococcosis: current problems and surgical treatment. Multimed Man Cardiothorac Surg 2017; in press. doi: 10.1510/mmcts.2017.017. 